BULl. Austral. MATH. SOC.

$05 C 99,05 C 35$

VOL. 36 (1987) $441-447$

\title{
ON MAXIMUM MATCHINGS IN CUBIC GRAPHS
}

\section{WITH A BOUNDED NUMBER OF BRIDGE-COVERING PATHS}

\author{
Gary Chartrand ${ }^{1}$, S.F. Kapoor, !rtrud R, Oellermann \\ ANid Sergio Ruiz ${ }^{2}$
}

It is proved that if $G$ is a connected cubic graph of order $p$ all of whose bridges lie on $r$ edge-disjoint paths of $G$, then every maximum matching of $G$ contains at least $p / 2-\lfloor 2 r / 3\rfloor$ edges. Moreover, this result is shown to be best possible.

1. Introduction and historical background

A matching in a graph $G$ is a set of pairwise nonadjacent (independent) edges of $G$. A matching with maximum cardinality is a maximum matching. If $G$ has order $p$, then a matching of cardinality $p / 2$ is called a perfect matching. Graphs with perfect matchings were characterized by Tutte [5].

Received 5 January 1987. ' ${ }^{1}$ Research partially supported by a Faculty Research Fellowship from Western Michigan University. ${ }^{2}$ Research supported by the United Nations through the program PNUD-UNESCO, and Universidad Catolica de Valparaiso (Chile).

Copyright Clearance Centre, Inc. Serial-fee code: 0004-9729/87 $\$ A 2.00+0.00$. 
442

G. Chartrand, S.F. Kapoor, O.R, Oellermann and S. Ruiz

THEOREM A. (Tutte). A graph $G$ has a perfect matching if and only if for every proper subset $S$ of $V(G)$, the number of odd components of $G-S$ does not exceed $|S|$.

Much research has centred around the determination of regular graphs that contain perfect matchings. A well known result on this subject is due to Petersen [4].

THEOREM B. (Petersen). Every cubic groph with at most two bridges contains a perfect matching.

This result cannot be improved, in general, since cubic graphs having three bridges but no perfect matchings exist. The graph of Figure 1 is the unique smallest such graph.

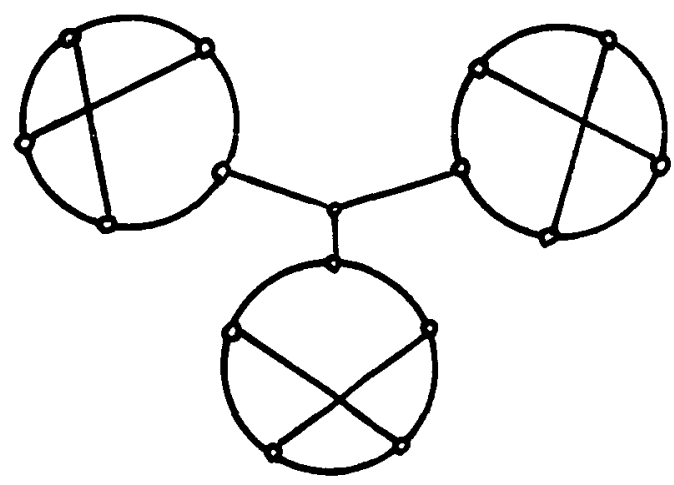

Figure 1

Note that the three bridges of the graph of Figure $I$ do not lie on a single path. Indeed, since this graph has no perfect matching, this property is necessary, by a result of Errera [3].

THEOREM C. (Errera). If all the bridges of a connected cubic groph $G$ lie on a single path of $G$, then $G$ has a perfect matching.

The goal of this paper is to provide a generalisation of Theorem $C$ by establishing a lower bound on the cardinality of a maximum matching in a connected cubic graph all of whose bridges lie on a specified number of edge-disjoint paths. Towards this end we state the following generalisation (see [1]) of the aforementioned theorem of Tutte. 
THEOREM D. Let $G$ be a cubic groph of order $p$ and let $\ell$ be an integer with $0 \leq \ell \leq p / 2$. Then every maximum matching of $G$ has at least $(p-2 l) / 2$ edges if and only if for every proper subset $S$ of $V(G)$, the number of odd components of $G-S$ does not exceed $|S|+2 l$.

\section{The main result}

We are now prepared to present a bound on the number of edges in a maximum matching in a connected cubic graph $G$ in terms of the number of paths containing the bridges of $G$.

THEOREM 1. If the bridges of a connected cubic graph $G$ lie on $r$ edge-disjoint paths of $G$, then each maximum matching of $G$ contains at least $p / 2-\lfloor 2 r / 3\rfloor$ edges.

Proof. Suppose, to the contrary, that $G$ contains a maximum matching $M$ with fewer that $p / 2-\lfloor 2 r / 3\rfloor$ edges. By Theorem $D$ there exists a proper subset $S$ of $V(G)$ such that the number $n$ of odd components of $G-S$ exceeds $|S|+2\lfloor 2 r / 3\rfloor$. Let $|S|=k$. Since $p$ is even, $n$ and $k$ are of the same parity, so that

$$
n \geq k+2\lfloor\underline{2 r} / 3\rfloor+2 . .
$$

Denote the odd components of $G-S$ by $G_{1}, G_{2}, \ldots, G_{n}$. Since $G$ is connected, every component $G_{i}(1 \leq i \leq n)$ contains at least one vertex that is adjacent to some vertex of $S$. Suppose, without loss of generality, that $G_{1}, G_{2}, \ldots, G_{t}$ denote the odd components of $G-S$ for which there exists exactly one edge $e_{i}$ joining a vertex in $G_{i}(1 \leq i \leq t)$ to a vertex of $S$. For $i=t+1, t+2, \ldots, n$, then, there are at least three edges joining vertices of $G_{i}$ to vertices of $S$; otherwise, for some $j(t+1 \leq j \leq n)$, vertices of $G_{j}$ are joined to vertices of $S$ by exactly two edges, implying that $G_{j}$ has an odd number of odd vertices, which is not possible.

Let $P_{1}, P_{2}, \ldots, P_{r}$ denote $r$ edge-disjoint paths of $G$ which contain all the bridges of $G$. Then for every $i(1 \leq i \leq r)$, at most two bridges of $G$ that lie on $P_{i}$ are in the set $\left\{e_{1}, e_{2}, \ldots, e_{t}\right\}$. Hence $t \leq 2 r$. Since at least $t+3(n-t)=3 n-2 t$ edges join vertices of 
G. Chartrand, S.F. Kapoor, O.R. Oellermann and S. Ruiz

$V\left(G_{1}\right) \cup V\left(G_{2}\right) \cup \ldots \cup V\left(G_{n}\right)$ to vertices of $S$ it follows that $3 n-4 r \leq 3 n-2 t \leq 3 k$. Therefore, $3(n-k) \leq 4 r$ so that by $(*)$, $3(2|2 r / 3|+2) \leq 4 r$, that is $3\lfloor 2 r / 3 \mid+3 \leq 2 r$. However,

$$
2 r+1=3((2 r-2) / 3)+3 \leq 3\lfloor 2 r / 3\rfloor+3 \leq 2 r,
$$

which gives a contradiction.

Another bound (see [2]) for the number of edges in a maximum matching in a connected cubic graph $G$ depends only on the number of bridges in $G$.

THEOREM E. Every maximom matching in a connected cubic graph of order $p$ with fewer than $3(l+1)$ bridges $(l \geq 0)$ has at least $(p-2 \ell) / 2$ edges.

If the bridges of a connected cubic graph lie on sufficiently few paths, then the bound provided in Theorem 1 on the number of edges in a maximum matching is an improvement on the bound provided in Theorem $\mathrm{E}$. A specific statement of this improved result is given next.

COROLLARY 1. Let $G$ be a connected cubic groph of order $p$ having $m$ bridges, and let. $\ell \geq 0$ be an integer such that $3 l \leq m<3(\ell+1)$. If these bridges lie on $r$ edge-disjoint paths, where $\lfloor 2 r / 3\rfloor<\ell$, then the number of edges in a maximum matching of $G$ is at least $p / 2-\lfloor 2 r / 3\rfloor$.

The result in Corollary 1 can be shown to be sharp, which we do next. Since the case $\ell=0$ corresponds to the existence of at most 2 bridges in a connected cubic graph, and sharpness is already known, we consider $\ell \geq 1$ to be given, and choose the maximum $r$ with $r \equiv 0(\bmod 3)$, say $r=3 s$, such that $\lfloor 2 r / 3\rfloor<\ell$. Then

$$
r=\left\{\begin{array}{lll}
(3 \ell-6) / 2 & \text { if } \ell \text { is even. } \\
(3 \ell-3) / 2 \text { if } \ell \text { is odd. }
\end{array}\right.
$$

We show that there exists a connected cubic graph $G$ of order $p$ having $m=3 l+j$ bridges $(j=0,1,2)$ all of which lie on $r$ edge-disjoint paths but no fewer, such that each maximum matching contains $p / 2-\lfloor 2 r / 3\rfloor$ edges.

We begin by constructing a graph $P_{n}^{*}(n \geq 1)$, consisting of graphs $H_{1}, H_{2}, \ldots, H_{n}$, where $H_{i}(1 \leq i \leq n-1)$ is obtained by deleting an 
edge of $K_{4}$ and $H_{n}$ is obtained by subdividing an edge of $K_{4}$. Denote the two vertices of degree 2 in $H_{i}(1 \leq i \leq n-1)$ by $u_{i}$ and $v_{i}$ and the vertex of degree 2 in $H_{n}$ by $u_{n}$. Then $P_{n}^{*}$ is produced by joining $v_{i}$ and $u_{i+1}(1 \leq i \leq n-1)$. Observe that each $P_{n}^{*}(n \geq 1)$ has odd order. Let the graph $H$ be the $12 s$-cycle $w_{1}, w_{2}, \ldots, w_{12 s}, w_{1}$ to which we add $2 s$ new vertices $x_{1}, x_{2}, \ldots, x_{2 s}$, where $x_{i}$ is joined to $w_{6 i-5}, w_{6 i-3}$ and $w_{6 i-1}(1 \leq i \leq 2 s)$. Consider next the graphs $G_{1}, G_{2}, \ldots, G_{6 s-1}$, each isomorphic to $P_{1}^{*}$, and the graph $G_{6 s}$, where

$$
G_{6 s}=\left\{\begin{array}{lll}
P_{7+j}^{*} & \text { if } \ell \text { is even }, \\
P_{4+j}^{*} & \text { if } \ell \text { is odd . }
\end{array}\right.
$$

The desired graph $G$ is now produced by joining $w_{2 i}$ to the vertex $u_{1}$ in $G_{i}(1 \leq i \leq 6 s)$ by an edge $e_{i}$. Figure 2 illustrates the graph $G$ for $\ell=3, r=3, s=1, m=9$ and $j=0$.

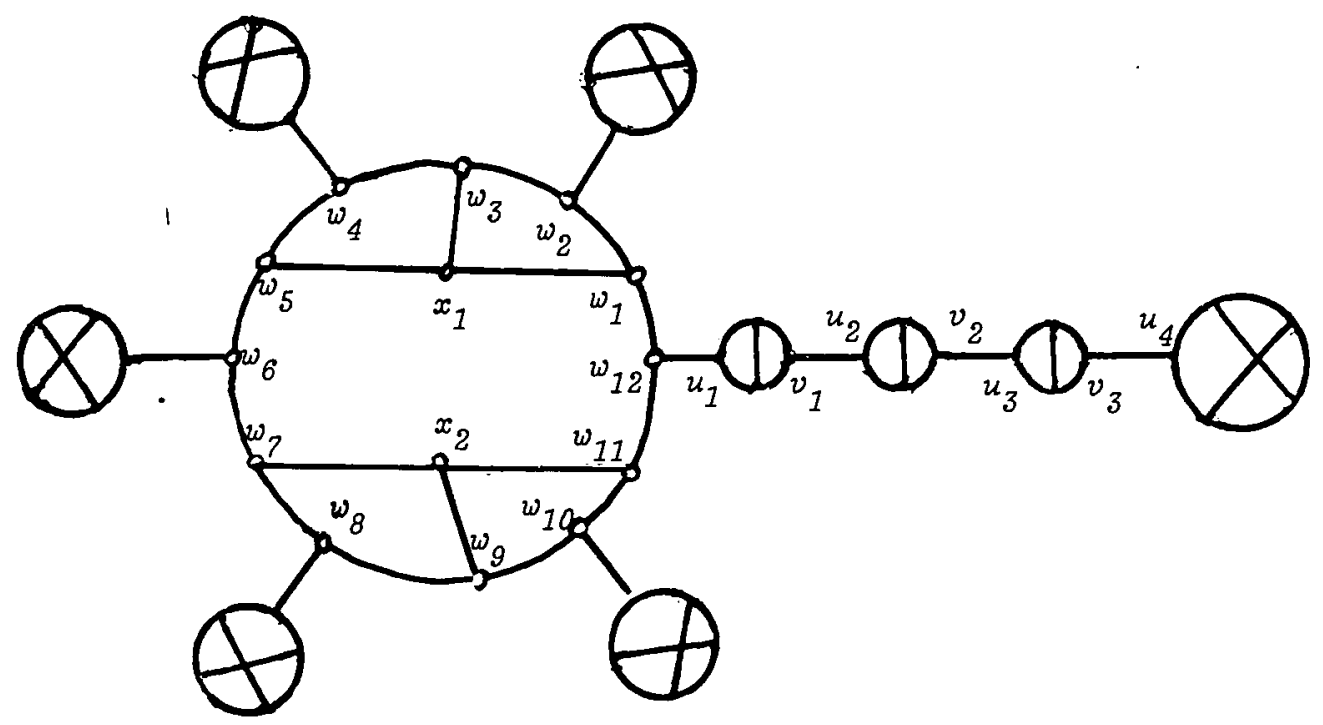

Figure 2 
clearly $G$ is connected, cubic, and each edge $e_{i}(1 \leq i \leq 68)$ is a bridge of $G$. Further, since $G_{68}$ contains $6+j$ or $3+j$ bridges, depending on whether $\ell$ is even or odd, respectively, it follows that $G$ contains exactly $6 s+6+j$ or $6 s+3+j$ bridges, according to whether $\ell$ is even or odd. Since the bridges of $G$ lie on $r=3 s$ edgedisjoint paths, Corollary 1 implies that every maximum matching of $G$ contains at least $p / 2-\lfloor 2 r / 3\rfloor$ edges.

It remains to be shown that every maximum matching of $G$ contains at most $p / 2-\lfloor 2 r / 3\rfloor$ edges. We use Theorem $D$ to prove this statement. Let $S=\left\{w_{2 i} \mid 1 \leq i \leq 6 s\right\} \cup\left\{x_{1}, x_{2}, \ldots, x_{2 s}\right\}$. Then $|S|=8 s$, and

$$
G-S=\left\{\begin{array}{l}
6 s K_{1} \cup(6 s-1) P_{1}^{*} \cup P_{7+j}^{*} \text { if } \ell \text { is even, } \\
6 s K_{1} \cup(6 s-1) P_{1}^{*} \cup P_{4+j}^{*} \text { if } \ell \text { is odd. }
\end{array}\right.
$$

Therefore, $G-S$ contains $128=|S|+4 s$ odd components. Theorem D now implies that every maximum matching of $G$ contains at most $p / 2-2 s=p / 2-\lfloor 2 r / 3\rfloor$ edges. Hence every maximum matching of $G$ contains exactly $p / 2-\lfloor 2 r / 3 \mid$ edges.

The cases where $r \equiv 1(\bmod 3)$ or $r \equiv 2(\bmod 3)$ can be handled in a similar manner. If $r \equiv 1(\bmod 3)$, the maximum $r$ with $\left\lfloor 2 r / 3_{-} \mid<\ell\right.$ is given by

$$
r= \begin{cases}(3 \ell-4) / 2 & \text { if } \ell \text { is even, } \\ (3 \ell-1) / 2 & \text { if } \ell \text { is odd. }\end{cases}
$$

Further, the maximum $r$ for $r \equiv 2(\bmod 3)$ and $\lfloor 2 r / 3\rfloor<\&$ satiafies

$$
r= \begin{cases}(3 \ell-2) / 2 & \text { if } \ell \text { is even, } \\ (3 \ell+1) / 2 & \text { if } \ell \text { is odd. }\end{cases}
$$

Then using a construction similar to the one described for $r \equiv 0(\bmod 3)$ we can show, for the above choices of $r$, that there is a graph $G$ having $m+j$ bridges $(j=0,1,2$ and $3 \ell \leq m<3(\ell+1))$ all of which lie on $r$ edge-disjoint paths and where every maximum matching of $G$ has $p / 2-\lfloor 2 r / 3\rfloor$ edges. Consequently, the result stated in corollary 1 is the best possible. 


\section{References}

[1] c. Berge, "Two theorems in graph theory", Proc. Nat. Acad, Sci. 43 (1957), 842-844.

[2] G. Chartrand, S.F. Kapoor, L. Lesniak and S. Schuster, "Near 1factors in graphs", Proceedings of the Fourteenth Southeastern Conference on Combinatorics, Graph Theory and Computing. Congressus Nomerantium 41 (1984), 131-147.

[3] A. Errera, "Du colorage des cartes", Mathesis 36 (1922), 56-60.

[4] J. Petersen, "Die theorie der regulären graphen", Acta Math. 15 (1891), 163-220.

[5] W.T. Tutte, "The factorizations of linear graphs", J. London Math. Soc. 22 (1947), 107-111.

Department of Maths and Statistics

Western Michigan University

KALAMAZOO, MI 49008 - 3899

U.S.A.

Instituto de Matematicas, Universidad Catolica de Valpararaiso, Chile. 